Journal of Circuits, Systems, and Computers

(C) World Scientific Publishing Company

\title{
Mathematical description of differential equation solving electrical circuits
}

\author{
K. NAKKEERAN \\ School of Engineering, Fraser Noble Building, King's College, \\ University of Aberdeen, Aberdeen AB24 3UE, United Kingdom \\ K.Nakkeeran@abdn.ac.uk \\ Received (Day Month Year) \\ Revised (Day Month Year) \\ Accepted (Day Month Year)
}

\begin{abstract}
We show that the working principle of the differential equation solving analog electrical circuits is exactly the same as the Picard's method available for numerically solving the ordinary differential equations. The integrator circuit (low-pass filter) uses an initial condition and electrical input signal to generate the Maclaurin's series of a time varying function in recursion. This direct connection between the differential equation solving electrical circuits and Picard's method can be exploited to simplify the procedure of Picard's method to solve any order linear and nonlinear differential equations.
\end{abstract}

Keywords: Electric circuits; Ordinary differential equations (ODEs); Picard's method.

\section{Introduction}

The dynamics of any system is usually modeled using differential equations. Depending on the complexity of the system, the modeled differential equation will also be complex in nature. The types of the differential equations include linear and nonlinear ordinary and partial differential equations. Different procedures were developed to study and solve the differential equations. The studies on the differential equations help to control the systems and to explore the stability of the systems. On the other hand, the exact solution of the differential equations helps in understanding the complete dynamics of the systems. When the system equation becomes quite difficult to derive the exact analytical solution, the other powerful handy procedure namely the numerical methods are used to solve the initial value problems and boundary value problems.

Analog electric circuits can also solve the ordinary differential equations (ODEs). Basically a low-pass-filter with resistor, capacitor and operational amplifier is utilized to design a simple integrator circuit. ${ }^{1}$ For any given ODE, a proper combination of the integrators, adders and multipliers can be used to visualize the solution for the dependent variable on an oscilloscope, while time $(t)$ being the independent variable. In this work, we show that an analog electric circuit which can solve an 
ODE, works on the same basic concept of how Picard's method solves the ODE, numerically. In recursion, an integrator circuit uses the initial condition and the electrical signal input to generate the Maclaurin's series for the output integrated function. This direct connection between the differential equation solving electric circuits and Picard's method can be exploited to simplify the procedure of Picard's method to solve any order linear and nonlinear differential equations.

\section{Analog electric circuit ODE solver}

A real time analog integrator circuit can be designed with a resistor, capacitor and an operational amplifier as shown in Fig. 1. Figure 1 also shows the the conventional schematic representation of the integrator on the right-hand side. The time constant $R C$ can be scaled to ' 1 ' using a resistor value $R=1 \mathrm{M} \Omega$ and a capacitor value $C=1$ $\mu \mathrm{F}$. Hence if the electric signal input to the integrator is $y^{\prime}(t)\left({ }^{\prime}\right.$ denotes $\left.d / d t\right)$, then the output would be $y(t)$. In the integrator one can give the initial condition also as shown in the right-hand side of Fig. 1.

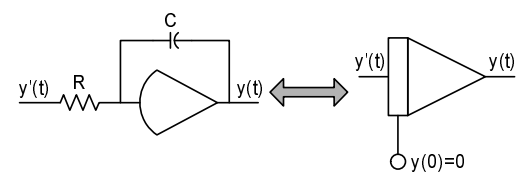

Fig. 1. Electrical integrator circuit (left-hand side) and its pictorial representation (right-hand side).

Now let us see how the integrator can be used to solve an ODE. Consider a simple ODE in the form:

$$
y^{\prime \prime}+y=0 .
$$

Let the initial conditions for the ODE (1) are $y^{\prime}(0)=1$ and $y(0)=0$. ODE (1) can be rewritten as $y^{\prime \prime}=-y$. This way of rewriting the ODE with the highest derivative in terms of the lower derivative terms will help to realize the analog circuit solver as shown in Fig. 2. From the expression $y^{\prime \prime}=-y$, it is obvious that the second derivative $\left(y^{\prime \prime}\right)$ potential is equal to the negative value of the solution $(y)$. That is why in the circuit shown in Fig. 2, the output of the inverter is fed back as the input of the first integrator. That is how the circuit becomes the solver for the ODE (1).

In fact the solution of the ODE (1) and its first derivative $y^{\prime}(t)$ take the analytic forms:

$$
y(t)=\sin (t), \quad y^{\prime}(t)=\cos (t) .
$$

Thus the $\sin (t)$ and $\cos (t)$ waveform can be visualized in the oscilloscope, respectively, at the output of the integrators two and one in Fig. 2. As such, the circuit 


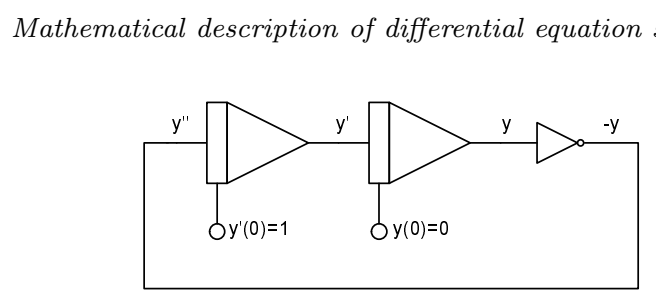

Fig. 2. Analog circuit solver for the ODE (1).

in Fig. 2 behaves like an oscillator. Let us see how these oscillatory waveforms are generated. The obvious source in the circuit is the initial condition $y^{\prime}(0)=1$. Hence when $t=0$ at the output of the first integrator, 1 is available for $y^{\prime}(t)$. Thus $y^{\prime}(0)=1$ will be the input to the second integrator which integrates $y^{\prime}(t)$ to get $y(t)$ as $t$. Then the inverter generates $-y$ which equals $y^{\prime \prime}$ as given by the ODE (1). This whole process can be treated as the first iteration:

\section{First iteration:}

$$
y^{\prime}=1 ; \quad y=t ; \quad y^{\prime \prime}=-t .
$$

Now, at the input of the first integrator, $-t$ is available as the electrical signal which will be integrated and added with the initial condition 1 to generate $y^{\prime}=$ $1-t^{2} / 2$. Thus the electrical signal $1-t^{2} / 2$ will be integrated by the second integrator to generate $y=t-y^{3} / 6$. Hence the second iteration is given by:

\section{Second iteration:}

$$
y^{\prime}=1-\frac{t^{2}}{2 !} ; \quad y=t-\frac{t^{3}}{3 !} ; \quad y^{\prime \prime}=-t+\frac{t^{3}}{3 !} .
$$

This process can go on indefinitely within no time to generate the following $n^{\text {th }}$ iteration:

$n^{\text {th }}$ iteration:

$$
\begin{gathered}
y^{\prime}=1-\frac{t^{2}}{2 !}+\frac{t^{4}}{4 !}-\frac{t^{6}}{6 !}+\cdots \\
y=t-\frac{t^{3}}{3 !}+\frac{t^{5}}{5 !}-\frac{t^{7}}{7 !}+\cdots \\
y^{\prime \prime}=-t+\frac{t^{3}}{3 !}-\frac{t^{5}}{5 !}+\frac{t^{7}}{7 !}-\cdots
\end{gathered}
$$

From the above expressions for $y, y^{\prime}$ and $y^{\prime \prime}$ it is obvious that the iteration process, in fact, generates the next term in the Maclaurin's series corresponding to the analytical expression for $y=\sin (t), y^{\prime}=\cos (t)$ and $y^{\prime \prime}=-\sin (t)$, respectively. 


\section{Picard's method for differential equations}

We now consider Picard's method for solving differential equations. Starting with the initial value problem, we have,

$$
y^{\prime}=f(t, y), \quad \text { with } \quad y(0)=y_{0} .
$$

Here we have chosen the initial point $t_{0}=0$ in equation (6) in order to simplify the calculations. If both $f(t, y)$ and $f_{y}(t, y)$ are continuous then a solution to equation (6) exists. $^{2}$

The following method was developed by Emile Picard in $1891 .^{3}$ If $y(t)$ is the solution of equation (6), then by reformulating equation (6) as an equivalent integral equation,

$$
\int_{0}^{t} y^{\prime}(z) d z=\int_{0}^{t} f(z, y(z)) d z .
$$

The Fundamental Theorem of Calculus is used to integrate the left-hand side of equation (7), and the result, after rearranging the terms, is:

$$
y(t)=y_{0}+\int_{0}^{t} f(z, y(z)) d z .
$$

If we use equation (8) and input a formula for $y(z)$ in the integrand $f(z, y(z))$, then the function $y(t)$ on the left-hand side is considered as the output. Start by substituting $Y_{0}(z)=y_{0}$ for $y(z)$ in $f(z, y(z))$ and get the integrand $f\left(z, y_{0}\right)$, this defines $Y_{1}(z)$. Next substitute $Y_{1}(z)$ for $y(z)$ and get the integrand $f\left(z, Y_{1}(z)\right)$, this will define $Y_{2}(z)$, etc.,. The process is repeated, and eventually leads to a sequence of functions $\left\{Y_{n}(z)\right\}$ which are defined successively by:

$$
Y_{n+1}(t)=y_{0}+\int_{0}^{t} f\left(z, Y_{n}(z)\right) d z \text { for } n \geq 0
$$

The sequence of approximations converges to the solution, i.e.,

$$
\lim _{n \rightarrow \infty} Y_{n}(t)=y(t) .
$$

Consider the following initial value problem:

$$
y^{\prime}=y-t \quad \text { with } \quad y(0)=2 .
$$

When we use the Picard's iterative method to find the solution of the equation (11) up to six iterations (starting with $Y_{0}=2$ ), we get

$$
Y_{6}=2+2 t+\frac{t^{2}}{2}+\frac{t^{3}}{6}+\frac{t^{4}}{24}+\frac{t^{5}}{120}
$$


Techniques from calculus can be used to find the solution $y(t)=1+t+\exp (t)$. We can express this as a Maclaurin series and observe that the sequence $\left\{Y_{n}(t)\right\}$ converges to the solution

$$
y(t)=1+t+\exp (t)=2+2 t+\frac{t^{2}}{2 !}+\frac{t^{2}}{2 !}+\cdots+\frac{t^{n}}{n !}+\cdots .
$$

\section{Extension to higher order differential equations}

Let us see how to solve the initial value problem for a second order differential equation using Picard's iterative method. Any second order ODE can be transformed to a coupled system of two first order ODEs. Thus we have to consider the following system of ODEs to find the solution of a second order ODE using Picard's method:

$$
\begin{aligned}
& x^{\prime}=f(t, x, y) \text { with } x(0)=x_{0}, \text { and } \\
& y^{\prime}=g(t, x, y) \text { with } y(0)=y_{0},
\end{aligned}
$$

Picard's iteration can be used to generate sequences $\left\{X_{n}(t)\right\}$ and $\left\{Y_{n}(t)\right\}$ which converge to the solution functions $x(t)$ and $y(t)$, respectively. ${ }^{4}$ They are defined by

$$
\begin{gathered}
X_{0}(t)=x_{0}, \\
Y_{0}(t)=y_{0}, \\
X_{n+1}(t)=x_{0}+\int_{0}^{t} f\left(z, X_{n}(z), Y_{n}(z)\right) d z \text { for } n \geq 0, \\
Y_{n+1}(t)=y_{0}+\int_{0}^{t} g\left(z, X_{n}(z), Y_{n}(z)\right) d z \text { for } n \geq 0 .
\end{gathered}
$$

The sequences of approximations converge to the solution, i.e.,

$$
\begin{aligned}
& \lim _{n \rightarrow \infty} X_{n}(t)=x(t) \text { and } \\
& \lim _{n \rightarrow \infty} Y_{n}(t)=y(t) .
\end{aligned}
$$

Consider the following second order ODE with the initial conditions:

$$
y^{\prime \prime}=3 y^{\prime}-2 y, \quad \text { with } \quad y^{\prime}(0)=5 \text { and } y(0)=2 .
$$

Above second order ODE (18) can be transformed to a coupled system of two first order ODEs as follows:

$$
\begin{aligned}
& x^{\prime}=-x-2 y \text { with } x(0)=-1, \text { and } \\
& y^{\prime}=3 x+4 y \text { with } y(0)=2 .
\end{aligned}
$$

Using the Picard's iterative method the solutions of the equations (19) up to five iterations (starting with $X_{0}=-1$ and $Y_{0}=2$ ) are found to be 
$6 \quad$ K. Nakkeeran

$$
\begin{aligned}
& X_{5}=-1-3 t-\frac{7}{2} t^{2}-\frac{5}{2} t^{3}-\frac{31}{24} t^{4}-\frac{21}{40} t^{5} \\
& Y_{5}=2+5 t+\frac{11}{2} t^{2}+\frac{23}{6} t^{3}+\frac{47}{24} t^{4}+\frac{19}{24} t^{5}
\end{aligned}
$$

The analytic solutions are $x(t)=\exp (t)-2 \exp (2 t)$ and $y(t)=-\exp (t)+$ $3 \exp (2 t)$ and can be expanded in their respective Maclaurin series. Hence, we can observe that the sequences converge to the solution

$$
\begin{aligned}
x(t) & =\exp (t)-2 \exp (2 t) \\
& =-1-3 t-\frac{7}{2} t^{2}-\frac{5}{2} t^{3}-\frac{31}{24} t^{4}-\frac{21}{40} t^{5}-\frac{127}{720} t^{6}-\cdots, \\
y(t) & =-\exp (t)+3 \exp (2 t) \\
& =2+5 t+\frac{11}{2} t^{2}+\frac{23}{6} t^{3}+\frac{47}{24} t^{4}+\frac{19}{24} t^{5}+\frac{191}{720} t^{6}+\cdots .
\end{aligned}
$$

Thus any $n^{\text {th }}$ order ODE can be transformed to $n$-system of first order ODEs and then the Picard's method can be used to find the solutions.

\section{Discussion}

When we compare the mathematical description of how an analog electric ODE solver works (discussed in Section 2) and the Picard's method to solve the ODEs (discussed in Section 3), it is quite obvious that both of them are one and the same. One can clearly see how both of them generate the Maclaurin series as the solution for the ODE.

In Section 3, we have described how the higher order ODEs are solved using the Picard's iterative method. In fact there is no need to transform the higher order ODEs to the system of first order ODEs to use the Picard's method. When we use the schematic block diagram for the electric circuit ODE solver, any order ODE can be directly solved to generate the Maclaurin series. The electric circuit solver for the second order ODE (18) can be drawn schematically as shown in Fig. 3.

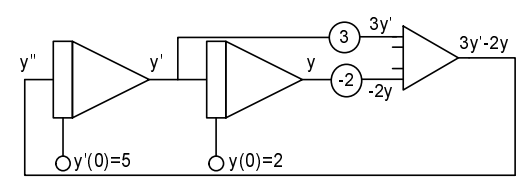

Fig. 3. Analog circuit solver for the second order ODE (18).

Now let us see how the solution for the second order ODE (18) is directly obtained from the electric circuit schematic diagram as shown in Fig. 3. When $t=0$, the 
initial conditions $y^{\prime}(0)=2$ and $y(0)=5$ are available, respectively, in the first and second integrators. These initial conditions are respectively, multiplied by 3 and -2 in the scalar multipliers and thus $3 y^{\prime}=15$ and $-2 y=-4$ are available as inputs to the summing amplifier. The output of the added $3 y^{\prime}-2 y=11$ is fed back as the input to the first integrator. Hence the output of the first integrator in addition to the initial condition would be $y^{\prime}=5+11 t$. Similarly, the output of the second integrator will be $y=2+5 t+11 t^{2} / 2$. This iteration process can be continued to generate the other terms in the Maclaurin series for $y(t)$ as in the equation (21).

The schematic block diagram can be used not only for first and second order linear ODEs, it can also be used for system of equations like higher order ODEs. Using multipliers and dividers in the block diagram, one can find the solutions for nonlinear ODEs as well. The examples illustrated in this work are all based on homogeneous ODEs. But the electric circuit can be constructed to solve nonhomogeneous ODEs and as well for time-varying ODEs. More details about how to draw the schematic diagrams for different kinds of ODEs can be found in the literature. ${ }^{1}$

\section{Conclusion}

In this work, we have shown the mathematical description of how an analog electric circuit ODE solver works. Both the Picard's iterative method for solving an ODE and the electric circuit solver generate the Maclaurin series of the solution of the ODE. The schematic block diagram representation of the ODE as an electric circuit can simplify the process of using the Picard's method for various kinds of ODEs. Picard's method is being utilized for solving various kinds of system equations. ${ }^{4,5,6,7,8,9,10}$ So we believe that the block diagram representation of the differential equations can simplify the procedure of applying the Picard's method to solve the equations.

\section{Acknowledgment}

Author wishes to thank Dr.K.Senthilnathan.

\section{References}

1. V. Rajaraman, Analog Computation and Simulation, (Printice Hall, New Delhi, 1971).

2. G. Birkhoff and G. C. Rota, Ordinary Differential Equations, (John Wiley and Sons, New York,1978).

3. (Charles) Emile Picard, Jour. de Math, 6 (1890) 145-210.

4. Einar Hille, Lectures on Ordinary Differential Equations, (Addison-Wesley Pub. Co., Reading, MA, 1969, pp. 32-41).

5. Stephen W. Goode, Differential Equations and Linear Algebra, (3rd edition, Prentice Hall, Upper Saddle River, NJ, 2005, Appendix 4).

6. R. Kent Nagle; Edward B. Saff; and Arthur David Snider, Fundamentals of Differential Equations and Boundary Value Problems, (4th edition, Addison-Wesley, Boston, MA, 2004, Chapter 13, Sections 1 and 2.) 
$8 \quad$ K. Nakkeeran

7. C. Henry Edwards; and David E. Penney, Differential Equations and Boundary Value Problems: Computing and Modeling, (3rd edition, Prentice Hall, Upper Saddle River, NJ, 2005, Appendix A.1.)

8. William E. Boyce; and Richard C. DiPrima, Elementary Differential Equations and Boundary Value Problems, (7th Edition, John Wiley and Sons, New York, NY, 2002, Chapter 2, Section 8.)

9. Garrett Birkhoff; and Gian-Carlo Rota, Ordinary Differential Equations, (4rd Ed., John Wiley and Sons, New York, NY, 1989, Chapter 1, p. 23.)

10. Arthur Wouk, On the Cauchy-Picard Method, The American Mathematical Monthly, 70, (1963), 158-162, Jstor. 